\title{
Sleep disturbance in primary brain tumor: prevalence, risk factors, and patient preferences
}

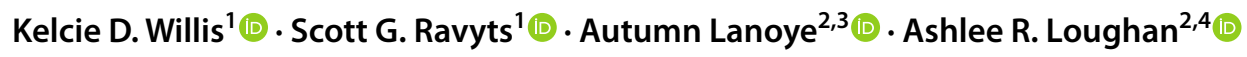

Received: 3 April 2021 / Accepted: 27 July 2021 / Published online: 9 August 2021

(c) The Author(s), under exclusive licence to Springer-Verlag GmbH Germany, part of Springer Nature 2021

\begin{abstract}
Purpose Primary brain tumor (PBT) patients report sleep disturbance due to their disease and treatment, yet few studies have utilized validated measures to understand the extent of patients' concerns and preferences for treatment. The purpose of this quality improvement project was to determine the prevalence and associated risk factors of sleep disturbance among PBT patients in our clinic and to evaluate interest in treatment for sleep disturbance.

Methods PBT patients completed validated measures of sleep disturbance and health during routine neuro-oncology visits. Patients also reported on sleep-related symptom management and their preferences for pharmacological and/or behavioral treatment.

Results Sleep disturbance was common, with $61.5 \%$ of PBT patients $\left(N=119 ; M_{\text {age }}=52.60\right.$ years; $50 \%$ male $)$ reporting poor sleep quality and $21.5 \%$ endorsing symptoms of insomnia. Insomnia could be explained by increased fatigue and corticosteroid use; sleep quality could be explained by fatigue. Patients in our clinic with higher grade tumors, significant sleep disturbance, of minority racial/ethnic status, and those not already taking sleep medications were more likely to report their symptoms and were not well-addressed by their medical team. Patients indicated a similar interest in pharmacological and behavioral treatment, warranting the availability of both in our clinic.

Conclusions Findings indicate a high prevalence of sleep disturbance in PBT patients, highlighting the need for increased screening, monitoring, and treatment in our neuro-oncology clinic. Future research would benefit from assessing the efficacy of behavioral treatments for sleep disturbance in this population.
\end{abstract}

Keywords Primary brain tumor $\cdot$ Sleep disturbance $\cdot$ Insomnia $\cdot$ Neuro-oncology $\cdot$ Psycho-oncology

\section{Introduction}

Sleep disturbance-including short sleep duration, poor quality of sleep, and sleep inefficiency - is one of the most common and consequential symptoms reported by cancer survivors [1]. As many as $60 \%$ of cancer patients report

Ashlee R. Loughan

ashlee.loughan@vcuhealth.org

1 Department of Psychology, Virginia Commonwealth University, Richmond, VA, USA

2 Massey Cancer Center, Virginia Commonwealth University, VA, Richmond, USA

3 Department of Health Behavior and Policy, School of Medicine, Virginia Commonwealth University, Richmond, VA, USA

4 Department of Neurology, School of Medicine, Virginia Commonwealth University, Richmond, VA, USA symptoms of sleep disturbance, a rate 2-3 times higher than that of the general population [2,3]. More recent studies suggest that nearly a third of cancer patients endorse severe insomnia with nearly two-thirds advocating for more consistent assessment of their sleep as part of their cancer care $[4,5]$. Among individuals with cancer, sub-optimal sleep is associated with a range of adverse physical, psychologi$\mathrm{cal}$, and functional consequences including increased pain, fatigue, and depression [6,7]. Despite the advent of safe and effective behavioral treatments for sleep disturbance [8], screening for sleep disorders in oncology settings is still limited, and pharmacological treatments remain the norm [9, $10]$.

Individuals with primary brain tumors (PBT) frequently report sleep disturbance and rate this problem as one of the most distressing symptoms $[11,12]$. One study reported that $38 \%$ of patients with PBT on steroids reported sleep disturbance [12]. Sleep disturbance is particularly problematic 
for this specific cancer population given their unique vulnerabilities: PBT patients are at a higher risk for cognitive impairment, and problems with sleep may magnify any neuropsychological deficits resulting from both the brain tumor and its treatment [13]. Furthermore, the side effects of prescribed sleep medications may also impair cognition and exacerbate PBT patients' risk of falls, fractures, and motor vehicle accidents [14]. Lastly, given that poor sleep quality is associated with higher levels of inflammatory biomarkers such as interleukin-6 (IL-6), prolonged difficulties with sleep may also increase PBT patients' risk for tumor progression and worsen their already poor prognoses $[15,16]$.

Despite the wide array of consequences associated with sleep disturbance in neuro-oncology, PBT patients have often been excluded from sleep research-perhaps, as reported in a review of PBT representation in quality of life research [17], due in part to presumed cognitive impairment. Two recent review articles $[11,18]$ highlight major drawbacks of extant research: first, the use of well-validated sleep measures is scarce, with past research predominantly relying on health-related quality of life measures or singleitem, self-report measures of sleep. Secondly, study samples were often small, composed of mixed brain cancer groups, or largely focused on patients with benign brain tumors in long-term remission from total tumor resection [11]. These methodological limitations inevitably obscure the prevalence of sleep disturbance in PBT and limit the generalizability of the existing knowledge base. To date, there is only one published study which directly analyzed the prevalence of sleep disturbance using validated sleep measures in a sample of Australian brain cancer patients (including both PBT and metastatic brain tumors) [19]. This study found that over half of the sample reported significant poor sleep quality, and $15 \%$ endorsed clinically significant symptoms of insomnia. Nevertheless, follow-up analyses with a sample of strictly PBT patients are needed to replicate these initial prevalence rates.

Information is also limited regarding potential risk factors for sleep disturbance among PBT patients, which might be useful in clarifying underlying mechanisms. In the larger sleep disturbance literature, certain demographic variables (e.g., female gender and advanced age) are known predisposing factors for developing insomnia [20], yet there has been no data to date that confirms this in neuro-oncology. Medical providers in neuro-oncology appear to attribute sleep disturbance solely to the use of corticosteroids and/or previous mood disorders [21]. However, Jeon and colleaugues [19] reported that other variables, such as participants' Karnofsky performance status (KPS) scores and levels of fatigue, were significant predictors of sleep quality in their mixed sample of primary and metastatic brain tumor patients. Other preliminary data in mixed neuro-oncology samples suggests radiation treatment, tumor bilaterality, and depressive symptoms may interfere with sleep [22, 23]. Further elucidating the etiology and underlying mechanisms of sleep disturbance in PBT are important steps in tailoring treatment to address these concerns.

Perhaps most importantly, existing research has not yet examined PBT patients' preferences for treatment or the extent to which they believe their reported symptoms are well-managed by their medical team. This information is especially important as a recent study found that only $20 \%$ of surveyed medical professionals in neuro-oncology routinely assessed for sleep disturbance, even though these providers perceived a high prevalence of sleep disturbance in their clinics [21]. This study also found that neuro-oncology providers were most likely to offer advice or prescribe medication to treat sleep disturbance, yet this study did not assess the patients' preferences for treatment, including their interest in non-pharmacological approaches (e.g., behavioral therapy). This is especially important as behavioral treatments have been shown to be highly effective [8] and are the preferred treatment option of other chronically ill samples who similarly have complex medication and treatment regimens [24]. Given the ubiquity and significance of sleeprelated concerns in this vulnerable population, a more thorough examination of the prevalence and risk factors for sleep disturbance-using comprehensive, validated sleep disturbance questionnaires - as well as PBT patients' beliefs and preferences for treatment is warranted. These inquiries will help inform the next steps of our neuro-oncology physicians in their treatment of sleep-related concerns.

The purpose of the present quality improvement project was threefold: (1) to determine the prevalence of sleep disturbance among PBT patients in our clinic, (2) to identify risk factors associated with sleep disturbance in our patient sample, and (3) to evaluate our PBT patients' beliefs about how well their medical team is currently managing their sleep-related symptoms and assess their interest in pharmacological and/or behavioral treatments for sleep disturbance. The prevalence of sleep disturbance was hypothesized to be comparable to that of previous research in neuro-oncology with fatigue, age, and corticosteroid use explaining greater sleep disturbance. We also hypothesized that a significant prevalence of sleep disturbance in our sample would translate into a strong belief that their symptoms are not wellmanaged by their medical team, especially considering recent research suggesting sleep disturbance is infrequently assessed in neuro-oncology. Finally, given what has been found in previous research with other medical populations [24], PBT patients were hypothesized to be most interested in behavioral over pharmacological treatments for sleep disturbance. 


\section{Methods}

\section{Design and study population}

Patients were asked to respond to an anonymous, voluntary quality improvement survey during their routine neurooncology visits at an urban National Cancer Institute-designated cancer center. Our IRB board ruled that our quality improvement survey did not necessitate review. Due to COVID-19 restrictions, visits occurred both in-person and via telehealth. During in-person visits, clinic nurses provided patients with the online Qualtrics survey to complete via iPad while waiting for care in private exam rooms. For telehealth appointments, patients were emailed a link to the survey to complete at home. Patients were required to be adults $(+18)$, diagnosed with a PBT, and literate in English to participate.

\section{Measures}

Demographic and clinical factors Patients self-reported demographic (e.g., age, gender, race/ethnicity) and clinical characteristics (e.g., tumor type, tumor grade, time since diagnosis, and corticosteroid medication use). Tumor type was categorized as glioblastoma, astrocytoma, oligodendroglioma, meningioma, or "other." Tumor grade was categorized dichotomously as low (grades I and II) or high (grades III and IV). Time since diagnosis was investigated as date of survey completion minus date of diagnosis, rounded to the nearest month. Current corticosteroid medication use was categorized dichotomously as either yes or no.

Insomnia Severity Index (ISI) [25] The ISI is a widely used screening measure of insomnia and has been validated in cancer populations [26]. Total scores on the ISI range from 0 to 28 , with higher scores corresponding to greater insomnia severity. The ISI has the following established cut-off scores: 0-7 (no clinically significant insomnia symptoms), 8-14 (subthreshold scores), 15-21 (clinical insomnia, moderate severity), and 22-28 (clinical insomnia, severe). Cronbach's alpha for the ISI in the current sample was 0.90 .

Pittsburgh Sleep Quality Index (PSQI) [27] The PSQI is a 19-item measure which assesses past-month sleep quality and produces seven component scores ranging from 0 to 3 , with higher scores corresponding to poorer sleep quality. Components include subjective sleep quality, sleep onset latency, sleep duration, sleep efficiency [ $\%$ of time spent sleeping / time spent in bed) $\times 100$ ], sleep disturbances, sleep medication use, and daytime dysfunction. Component scores are totaled to produce a global score ranging from 0 to 21 , with scores above 5 indicative of poor sleep quality [27]. Cronbach's alpha for PSQI in the current sample was 0.72 .

Brief Fatigue Inventory (BFI) [28] One item from the BFI was used to determine participants' level of fatigue during the past month on a scale from 0 (no fatigue) to 10 (as bad as you can imagine). The use of a single item for assessing fatigue is consistent with previous research [29, 30], though these studies did not use single items from the BFI specifically.

Napping A single-item asked patients to report the average number of hours per day spent napping in the past month.

Patient beliefs and interests Patients were also asked to report on the following: (1) their beliefs regarding how well their symptoms of insomnia were managed by their medical team and (2) their interest in insomnia treatment (i.e., pharmacological and/or behavioral treatment). Insomnia management was rated from 0 (not at all well-managed) to 10 (very well-managed); treatment interests were rated from 0 (strongly dislike) to 10 (strong prefer). For each of these questions, participants could also mark not applicable (i.e., no insomnia symptoms). See Table 1 for researcherdeveloped questions.

Table 1 Researcher developed questions assessing patients' beliefs of symptom management and patients' interests in treatment

\begin{tabular}{|c|c|}
\hline Patient beliefs item & Rating scale \\
\hline $\begin{array}{l}\text { 1. To what extent do you believe your insomnia symptoms are well-managed by your medical } \\
\text { providers? }\end{array}$ & $\begin{array}{l}0 \text { (not at all well-managed) to } 10 \text { (very } \\
\text { well-managed) }\end{array}$ \\
\hline Patient treatment interests items & Rating scale \\
\hline $\begin{array}{l}\text { 1. Insomnia can be treated with medications and/or techniques aimed at changing behaviors and } \\
\text { lifestyle factors. How would you describe your feelings towards medication treatment? }\end{array}$ & 0 (strongly dislike) to 10 (strongly prefer) \\
\hline $\begin{array}{l}\text { 2. Insomnia can be treated with medications and/or techniques aimed at changing behaviors and } \\
\text { lifestyle factors. How would you describe your feelings towards behavioral treatment? }\end{array}$ & 0 (strongly dislike) to 10 (strongly prefer) \\
\hline
\end{tabular}

Note: For each item, participants could also mark not applicable/no insomnia symptoms 


\section{Data analytic plan}

Descriptive analyses were computed to characterize the sample and estimate the prevalence of sleep disturbance. Patients with clinically elevated insomnia symptoms (ISI $\geq 15$ ) or poor sleep quality (PSQI $>5$ ) were categorized as experiencing sleep disturbance. Two logistic regressions were performed to identify demographic and clinical factors associated with sleep disturbance; variables included age, gender, race/ethnicity, time since diagnosis, tumor type, tumor grade, corticosteroid medication use, and fatigue severity. Patients' beliefs about how well their symptoms were managed by their medical team and their treatment interests (i.e., pharmacological and/or behavioral treatment) were assessed using frequencies. Patients' beliefs and interests were collapsed into two categorical responses, where responses of $0-4$ were negative/ low and responses of 6-10 were positive/high. Lastly, we used both one-way ANOVA and Pearson correlations to determine how these beliefs and interests differed across demographic (e.g., age, gender, race/ethnicity) and clinical (e.g., time since diagnosis, tumor type, tumor grade, corticosteroid medication use, sleep medication use, fatigue severity, ISI total, and PSQI total) variables.

\section{Results}

\section{Patient characteristics}

A total of 121 PBT patients participated in the survey. Two patients did not complete both the ISI and PSQI and thus were excluded, yielding a sample size of 119 for analysis. Patients were predominantly male (50.4\%), White (74.4\%), and middle-aged $(M=52.60$ years; $S D=15.39$; range, 21-83 years). Most patients reported high-grade brain tumor (61.3\%), and glioblastoma multiforme (GBM) was the most commonly reported tumor type (37\%). The average time since PBT diagnosis was 65.08 months $(S D=72.06$; range, 1-367 months). One fifth of patients (20.2\%) reported that they were currently prescribed corticosteroids. Finally, the patients' mean fatigue level during the previous two weeks was $4.39(S D=2.71$; range, $0-10)$. See Table 2 for complete participant demographics and clinical characteristics.

\section{Prevalence of sleep disturbance}

In terms of insomnia, patients' average score on the ISI was within the sub-clinical range $(M=8.81, S D=6.69)$. Based on established cut-offs, the breakdown of scores on the ISI was as follows: $44.6 \%$ no clinically significant insomnia,
$31.4 \%$ sub-clinical symptoms, $17.4 \%$ moderate symptoms, and $4.1 \%$ severe symptoms.

In terms of sleep quality, over half (61.5\%) of patients had poor sleep quality based on the PSQI total score ( $>5)$. Average sleep duration was $8.58 \mathrm{~h} / \mathrm{night}(S D=1.95 \mathrm{~h} / \mathrm{night})$, with $52.1 \%$ of participants reporting a sleep efficiency score below $85 \%$, a common cut-off indicative of poor sleep quality.

Participants with insomnia as measured by the ISI all endorsed poor sleep quality (PSQI $>5$ ), while $34.7 \%$ of participants who endorsed poor sleep quality (PSQI $>5$ ) also scored above the cut-off for insomnia (ISI $\geq 15$ ).

Sleep medication use was also prevalent, with $24 \%$ of participants using over-the-counter medication and $12.4 \%$ taking prescription sleep medication. Based on the ISI, insomnia severity for participants taking any kind of sleep medication $(M=11.90, S D=6.72)$ was nearly twice as high as those not taking medication $(M=6.46, S D=5.69$; $F(1,116)=22.63, p<0.001)$. Moreover, of those taking sleep medication, $62.7 \%$ screened positive for insomnia on the ISI.

Finally, daytime napping was common (69.7\%) with an average of $1.41 \mathrm{~h}(S D=1.41$; range, $0-6 \mathrm{~h})$ of reported daytime sleep per day.

\section{Risk factors of sleep disturbance}

Two logistic regressions were used to determine risk factors for sleep disturbance and insomnia, using the PSQI and ISI as outcome variables. In the first logistic regression, explaining insomnia correctly classified $82.8 \%$ of participants (sensitivity $=50 \%$; specificity $=92.2 \%$ ). Nagelkerke $R^{2}$ suggested that the model accounted for approximately $52.5 \%$ of the variance in insomnia. Specifically, corticosteroid medication use $(O R=5.97 ; \mathrm{CI}, 1.15,31.07)$ and fatigue severity $(O R=1.66 ; \mathrm{CI}, 1.23,2.25)$ were each found to significantly increase the likelihood of insomnia.

By contrast, the second logistic model explained $29.4 \%$ of the variance in sleep quality and correctly classified $71.9 \%$ of participant's sleep quality (sensitivity $=50 \%$; specificity $=84.7 \%)$. Only fatigue severity explained the presence of poor sleep quality $(O R=1.29 ; \mathrm{CI}, 1.07,1.56)$. Supplemental Table 1 provides the full results of the logistic regressions.

\section{Patients' beliefs about management of sleep-related symptoms}

Of those who positively screened for insomnia (ISI $\geq 15$ ), most patients $(64 \%)$ reported these symptoms were not well-managed (rating of 0-4) by their medical team, and only $24 \%$ reported that these symptoms were indeed wellmanaged (rating of $6-10 ; M=3.64 ; S D=2.46$ ). Similarly, in patients who reported clinically significant sleep disturbance (PSQI > 5), more patients indicated their symptoms were 
Table 2 Demographic and clinical characteristics of primary brain tumor sample $(N=119)$

\begin{tabular}{|c|c|c|c|c|}
\hline Variable & & & Frequency & Mean/percent (SD) \\
\hline Age & & & 119 & $\begin{array}{l}52.60 \text { years } \\
\text { (range } 21-83 \text { years) }\end{array}$ \\
\hline \multirow[t]{2}{*}{ Gender } & Male & & 60 & 50.4 \\
\hline & Female & & 58 & 48.7 \\
\hline \multirow[t]{3}{*}{ Race } & White & & 88 & 73.9 \\
\hline & African American & & 23 & 19.3 \\
\hline & Other & & 8 & 6.6 \\
\hline \multirow[t]{12}{*}{ Tumor type } & Meningioma & & 18 & 15.1 \\
\hline & & Grade I & 8 & 6.7 \\
\hline & & Grade II & 8 & 6.7 \\
\hline & Astrocytoma & & 27 & 22.7 \\
\hline & & Grade II & 13 & 10.9 \\
\hline & & Grade III & 13 & 10.9 \\
\hline & Oligodendroglioma & & 21 & 17.6 \\
\hline & & Grade II & 5 & 4.2 \\
\hline & & Grade III & 12 & 10.1 \\
\hline & Glioblastoma multiforme & & & \\
\hline & & Grade IV & 44 & 37.0 \\
\hline & Other/unknown & & 9 & 7.6 \\
\hline \multirow[t]{2}{*}{ Tumor grade } & Low & Grades I and II & 35 & 21.8 \\
\hline & High & Grades III and IV & 73 & 61.3 \\
\hline \multirow[t]{6}{*}{ Time since diagnosis } & & & 119 & 65.08 mos. (72.06) \\
\hline & & $>1$ year & 20 & 16.8 \\
\hline & & $1-3$ years & 43 & 36.0 \\
\hline & & $3-5$ years & 17 & 14.0 \\
\hline & & $5-10$ years & 13 & 10.4 \\
\hline & & $10+$ years & 25 & 20.4 \\
\hline \multicolumn{5}{|l|}{ Corticosteroid Rx } \\
\hline & Yes & & 24 & 20.2 \\
\hline & No & & 91 & 76.5 \\
\hline & Unsure & & 4 & 3.4 \\
\hline \multirow[t]{4}{*}{ Sleep medication } & No & & 68 & 57.1 \\
\hline & Yes & & 51 & 42.9 \\
\hline & & Prescribed & 15 & 12.6 \\
\hline & & Over-the-counter & 29 & 24.4 \\
\hline ISI total & & & 118 & $8.81(6.70)$ \\
\hline PSQI total & & & 117 & $7.19(4.27)$ \\
\hline
\end{tabular}

Note: ISI, Insomnia Severity Index; PSQI, Pittsburgh Sleep Quality Inventory not well-managed $(48.3 \%)$ than managed $(38.3 \% ; M=4.48$; $S D=3.37$ ).

Using both one-way ANOVA and Pearson correlations, we determined whether any demographic or clinical characteristics were related to patients' beliefs regarding how well their symptoms were managed. The results revealed that patients were more likely to believe their symptoms were not well-managed if they had higher grade tumors $(F(1,57)=6.75, p=0.01)$, were currently prescribed corticosteroids $(F(1,61)=4.70, p=0.03)$, were of a minority racial/ethnic background $(F(1,62)=4.61, p=0.04)$, were not already taking a prescription sleep medication, $(F(1$, $32)=4.55, p=0.04)$, or had higher ISI scores $(r=-0.35$, $p<0.01)$. No other demographic or clinical variables were significantly associated ( $p s>0.05)$.

\section{Patients' interests in treatment}

Of those who positively screened for insomnia (ISI $\geq 15$ ), $48 \%$ reported interest in pharmacological treatment and $41.7 \%$ in behavioral treatment. Of those who positively screened for sleep disturbance (PSQI $>5$ ), $40.5 \%$ reported 
interest in pharmacological treatment and 38.5\% in behavioral treatment.

Using both one-way ANOVA and Pearson correlations, we assessed whether any demographic or clinical variables determined an interest in pharmacological and/or behavioral treatment. Patients with poorer sleep quality as measured by the PSQI $(r=0.34, p<0.01)$ and patients who were already taking sleep medications $(F(1,75)=5.11, p=0.03)$ were more likely to rate pharmacological treatment more highly; no other demographic or clinical variables were found to be significantly related, $p s>0.05$.

\section{Discussion}

This study sought to better understand the prevalence and risk factors of sleep disturbance in a sample of PBT patients receiving care at a National Cancer Institute (NCI)designated neuro-oncology clinic. This data represents the first known investigation to use validated questionnaires of insomnia and sleep disturbance (i.e., Insomnia Severity Index and Pittsburgh Sleep Quality Index) in exclusively PBT patients, a sample with myriad vulnerabilities to sleep disturbance and its treatment. We found that over half of patients reported poor sleep quality and over a fifth endorsed moderate-to-severe symptoms of insomnia. Increased fatigue was found to explain both insomnia and sleep disturbance, whereas corticosteroid use was found to be only an indicator of insomnia. Our investigation also had the unique goal of elucidating patients' beliefs on how well these symptoms were being managed by their medical team as well as patients' interests in treatment moving forward. Those experiencing sleep disturbance did not feel their symptoms were properly addressed by their medical team, yet there was a similar interest in both pharmacological and behavioral treatments across the board. These findings suggest that our patients may potentially benefit from (1) additional screening of insomnia by medical staff and (2) behavioral interventions for insomnia by one of our two staff psychologists or our clinical psychology practicum students. Further, the results may prompt and guide other neuro-oncology clinics to enhance their care of sleep disturbance in their own patient populations.

The prevalence of insomnia in our sample was comparable to a recent study which examined sleep among patients with either primary or metastatic brain tumors [19] but lower than a prior study of patients with recurrent gliomas where insomnia was screened using an adverse event scale [31]. One possible reason for this discrepancy may be the relatively higher rate of sleep medication use in our sample, which may mask some of the symptoms of sleep disturbance. One study found that hypnotic medication might be helpful to improve sleep, mood, and cognitive functioning for patients with brain tumors [32]. The risk factors for sleep disturbance among our patients were consistent with past research. For example, one study found that $89 \%$ of patients with PBT who were categorized as having insomnia endorsed fatigue $[31,33]$. These findings are clinically relevant given that fatigue is known to adversely affect both the quality of life (e.g., disrupting mood) and functioning of PBT patients [34, 35]. Similarly, the association between corticosteroids use and sleep disturbance has been shown in previous investigations [31]. While previous research suggests that certain demographic variables [36], such as female gender or advanced age, predispose one to experience insomnia, this was not the case among our patients. However, the lack of an association between these demographic variables and sleep disturbance is consistent with other research examining sleep in patients with PBT $[19,31]$.

Given that previous findings have uncovered infrequent assessment of sleep disturbance in neuro-oncology [21], it is perhaps not surprising our data revealed a high rate of dissatisfaction with how well their sleep-related symptoms were managed by their medical team. Indeed, most of our patients with clinically significant sleep disturbance indicated that their symptoms were not properly addressed by their medical team. To manage the tumor and its side effects (e.g., edema), neuro-oncologists often prescribe medications and treatments that may negatively impact sleep (e.g., corticosteroids, radiation therapy). Thus, frequent assessment and routine monitoring of sleep disturbance is warranted, especially in those with higher grade tumors, those of a minority racial/ethnic background, those prescribed corticosteroids, and those not already taking sleep medications, as these individuals in our clinic were less likely to believe their symptoms were being addressed. Validated, brief instruments such as the ISI and PSQI may be two possible, practical additions to improve assessment in our NCI-designated, fastpaced, neuro-oncology clinic. Both measures may also assist with monitoring symptoms across the disease trajectory as they are sensitive to therapeutic change [26].

Increasing the identification of sleep disturbance is merely the first step in improving quality of life of PBT patients; patients will also need an appropriate treatment. Our findings indicate that patients in our clinic have a similar interest in both pharmacological and behavioral treatment for insomnia, regardless of whether they have clinically significant insomnia. Only those with poor sleep quality and those already taking sleep medications indicated a higher interest in medication as treatment. Previous findings suggest that pharmacological treatment is the norm in neurooncology [9, 21, 37], and many of our patients reported using an over-the-counter or prescription sleep medication. Of note, however, the majority of those using sleep medications still screened positive for insomnia-perhaps suggesting the need for combined pharmacological and behavioral 
treatment for these patients. While behavioral treatments are less likely to be recommended by individual medical providers [21], cognitive behavioral therapy for insomnia (CBT-I) is the recommended frontline treatment for insomnia by the American College of Physicians [8] and American Academy of Sleep Medicine [38]. CBT-I specifically targets dysfunctional sleep routines and attitudes without the use of medication. Many chronically ill patients prefer behavioral treatment such as CBT-I over medication given the risk of polypharmacy [24]; this is especially relevant to PBT patients who are often prescribed a variety of medications, including psychostimulants [39]. While CBT-I has been found to be efficacious in improving sleep disturbance in other cancer populations [37], PBT patients have been historically excluded from these trials. Overall, the significant proportion of interest in behavioral treatment identified in our sample warrants the availability of such treatments moving forward, though future investigations of CBT-I in PBT are also needed.

\section{Limitations}

The present study is not without limitations. First, the crosssectional design precludes our ability to establish temporal relationships between clinical risk factors and sleep disturbance in PBT patients. Secondly, though the use of well-established measures of sleep disturbance is a strength relative to past research, self-report measures are subject to reporting bias. Future research would also benefit from comprehensive scales of fatigue. Finally, given that these findings are based on a quality improvement project, additional clinical factors of interest, such as emotional distress, history of previous treatments, or functional status, were not assessed. Future research would benefit from examining a larger population and a broader range of relevant clinical variables to determine their association to sleep among this patient population.

\section{Conclusion}

Sleep disturbance and insomnia are highly prevalent among patients in our clinic with PBT. Patients with poor sleep are dissatisfied with the management of their symptoms and report an openness to either pharmacological or behavioral interventions for insomnia. These findings underscore the need for better screening of sleep disturbance by healthcare providers in our clinic and for future research to assess the efficacy of both pharmacological and behavioral interventions.
Supplementary Information The online version contains supplementary material available at https://doi.org/10.1007/s00520-021-06476-3.

Acknowledgements Thank you to all our patients. We would also like to thank our clinical nurse Joany Flynn for her contribution to this project.

Author contribution All authors contributed to the study conception and design. Material preparation, data collection, and analysis were performed by Kelcie D. Willis and Scott G. Ravyts. The first draft of the manuscript was written by both Kelcie D. Willis and Scott G. Ravyts, and all authors commented on previous versions of the manuscript. All authors read and approved the final manuscript.

Funding The project described was supported by CTSA award No. KL2TR002648 from the National Center for Advancing Translational Sciences. Its contents are solely the responsibility of the authors and do not necessarily represent the official views of the National Institutes of Health.

Data availability Data are available upon reasonable request from the corresponding author.

Code availability Not applicable.

\section{Declarations}

Ethics approval This is a quality improvement, needs assessment study. The Institutional Review Board at VCU has confirmed that no ethical approval is required as this data does not qualify as research and was collected for clinical purposes.

Consent to participate Not applicable.

Consent for publication Not applicable.

Conflict of interest The authors declare no competing interests.

\section{References}

1. Savard J, Savard M-H (2013) Insomnia and cancer prevalence, nature, and nonpharmacologic treatment prevalence and evolution of insomnia in patients with cancer. Clin Sleep Med 8:373-387. https://doi.org/10.1016/j.jsmc.2013.04.006

2. Savard J, Ivers H, Villa J, Caplette-Gingras A, Morin CM (2011) Natural course of insomnia comorbid with cancer: an 18-month longitudinal study. J Clin Oncol 29(26):3580-3586. https://doi. org/10.1200/JCO.2010.33.2247

3. Beck SL, Berger AM, Barsevick AM, Wong B, Stewart KA, Dudley WN (2010) Sleep quality after initial chemotherapy for breast cancer. Support Care Cancer 18(6):679-689. https://doi.org/10. 1007/s00520-009-0662-y

4. Nguyen-Michel V-H, Vecchierini M-F (2016) Exploration des troubles du sommeil chez le sujet âgé: quelles particularités? [Exploration of sleep disorders in the elderly: which particularities?]. Geriatr Psychol Neuropsychiatr Vieil 14(4):429-437

5. Harrold EC, Idris AF, Keegan NM et al (2020) Prevalence of insomnia in an oncology patient population: an Irish tertiary referral center experience. JNCCN J Natl Compr Cancer Netw 18(12):1623-1630. https://doi.org/10.6004/JNCCN.2020.7611

6. Loh KP, Zittel J, Kadambi S et al (2018) Elucidating the associations between sleep disturbance and depression, fatigue, and pain in older 
adults with cancer. J Geriatr Oncol 9(5):464-468. https://doi.org/10. 1016/j.jgo.2018.02.006

7. George GC, Iwuanyanwu EC, Anderson KO et al (2016) Sleep quality and its association with fatigue, symptom burden, and mood in patients with advanced cancer in a clinic for early-phase oncology clinical trials. Cancer 122(21):3401-3409. https://doi.org/10.1002/ cncr.30182

8. Qaseem A, Kansagara D, Forciea MA, Cooke M, Denberg TD (2016) Management of chronic insomnia disorder in adults: a clinical practice guideline from the american college of physicians. Ann Intern Med 165(2):125. https://doi.org/10.7326/M15-2175

9. Zhou ES, Partridge AH, Syrjala KL, Michaud AL, Recklitis CJ (2016) Evaluation and treatment of insomnia in adult cancersurvivorship programs. J Cancer Surviv 11(1):74-79. https://doi.org/10. 1007/s11764-016-0564-1

10. Garland SN, Barg FK, Cakouros B, Gehrman P, DuHamel KN, Mao JJ (2018) A qualitative examination of the factors related to the development and maintenance of insomnia in cancer survivors. Pall and Support Care 17(2):221-226. https://doi.org/10.1017/s1478 951518000135

11. Jeon MS, Dhillon HM, Agar MR (2017) Sleep disturbance of adults with a brain tumor and their family caregivers: a systematic review. Neuro Oncol 19(8):1035-1046. https://doi.org/10.1093/neuonc/ nox019

12. Armstrong TS, Ying Y, Wu J et al (2015) The relationship between corticosteroids and symptoms in patients with primary brain tumors: utility of the dexamethasone symptom questionnaire-chronic. Neuro Oncol 17(8):1114-1120. https://doi.org/10.1093/neuonc/nov054

13. Brownlow JA, Miller KE, Gehrman PR (2020) Insomnia and cognitive performance. Sleep Med Clin 15(1):71-76. https://doi.org/10. 1016/j.jsmc.2019.10.002

14. Proctor A, Bianchi MT (2012) Clinical pharmacology in sleep medicine. Int Sch Res Netw ISRN Pharmacol 2012. https://doi.org/10. 5402/2012/914168

15. Huang W-Y, Huang C-C, Chang C-C, Kor C-T, Chen T-Y, Wu H-M. Associations of self-reported sleep quality with circulating interferon gamma-inducible protein 10 , interleukin 6 , and high-sensitivity C-reactive protein in healthy menopausal women. Proost $\mathrm{P}$, ed. PLoS One. 2017;12(1):e0169216. https://doi.org/10.1371/journal. pone.0169216

16. Mullington JM, Simpson NS, Meier-Ewert HK, Haack M (2010) Sleep loss and inflammation. Best Pract Res Clin Endocrinol Metab 24(5):775-784. https://doi.org/10.1016/j.beem.2010.08.014

17. Loughan AR, Lanoye A, Aslanzadeh FJ et al (2019) Fear of cancer recurrence and death anxiety: Unaddressed concerns for adult neuro-oncology patients. Journal of Clin Psych in Med Settings 28(1):16-30. https://doi.org/10.1007/s10880-019-09690-8

18. Armstrong TS, Shade MY, Breton G et al (2017) Sleep-wake disturbance in patients with brain tumors. Neuro Oncol 19(3):323-335. https://doi.org/10.1093/neuonc/now119

19. Jeon MS, Dhillon HM, Koh E-S et al (2020) Exploring sleep disturbance among adults with primary or secondary malignant brain tumors and their caregivers. Neuro-Oncology Pract 8(1):48-59. https://doi.org/10.1093/nop/npaa057

20. Zhang B, Wing Y-K (2006) Sex differences in insomnia: a metaanalysis. Sleep 29(1):85-93. https://doi.org/10.1093/sleep/29.1.85

21. Jeon MS, Dhillon HM, Koh ES, Nowak AK, Hovey E, Agar MR (2020) Sleep disturbance in people with brain tumours and caregivers: a survey of healthcare professionals' views and current practice. Support Care Cancer 29(3):1497-1508. https://doi.org/10.1007/ s00520-020-05635-2

22. Powell C, Guerrero D, Sardell S et al (2011) Somnolence syndrome in patients receiving radical radiotherapy for primary brain tumours: a prospective study. Radiother Oncol 100(1):131-136. https://doi. org/10.1016/j.radonc.2011.06.028
23. Mainio A, Hakko H, Niemelä A, Koivukangas J, Räsänen P (2013) Insomnia among brain tumor patients: a population-based prospective study of tumor patients in Northern Finland. Journal of Psycho Onc 31(5):507-516. https://doi.org/10.1080/07347332.2013.822048

24. Azad N, Byszewski A, Sarazin FFA, McLean W, Koziarz P (2003) Hospitalized patients' preference in the treatment of insomnia: pharmacological versus non-pharmacological. Can J Clin Pharmacol 10(2):89-92

25. Bastien CH, Vallières A, Morin CM (2001) Validation of the insomnia severity index as an outcome measure for insomnia research. Sleep Med 2(4):297-307. https://doi.org/10.1016/S1389-9457(00) 00065-4

26. Savard M-H, Savard J, Simard S, Ivers H (2005) Empirical validation of the insomnia severity index in cancer patients. Psychooncology 14(6):429-441. https://doi.org/10.1002/pon.860

27. Buysse DJ, Reynolds CF, Monk TH, Berman SR, Kupfer DJ (1989) The Pittsburgh sleep quality index: a new instrument for psychiatric practice and research. Psychiatry Res 28(2):193-213. https://doi.org/ 10.1016/0165-1781(89)90047-4

28. Mendoza TR, Wang XS, Cleeland CS et al (1999) The rapid assessment of fatigue severity in cancer patients. Cancer 85(5):1186-1196. https://doi.org/10.1002/(SICI)1097-0142(19990301)85:5\%3c1186: AID-CNCR24\%3e3.0.CO;2-N

29. Kirsh KL, Passik S, Holtsclaw E, Donaghy K, Theobald D (2001) I get tired for no reason: a single item screening for cancer-related fatigue. J Pain Symptom Manag 22(5):931-937. https://doi.org/10. 1016/S0885-3924(01)00350-5

30. Van Hooff MLM, Geurts SAE, Kompier MAJ, Taris TW (2007) How fatigued do you currently feel? Convergent and discriminant validity of a single-item fatigue measure. J Occup Health 49(3):224 234. https://doi.org/10.1539/joh.49.224

31. Robertson ME, McSherry F, Herndon JE, Peters KB (2016) Insomnia and its associations in patients with recurrent glial neoplasms. Springerplus 5(1):1-5. https://doi.org/10.1186/s40064-016-2578-6

32. Chang MC, Chun MH (2020) The effect of hypnotics on sleep quality and cognitive function in patients with brain tumors. J Korean Neurosurg Soc 63(2):261-267. https://doi.org/10.3340/jkns.2019.0057

33. Fox SW, Lyon D, Farace E (2007) Symptom clusters in patients with high-grade glioma. J Nurs Scholarsh 39(1):61-67

34. Coomans MB, Dirven L, Aaronson NK et al (2019) Symptom clusters in newly diagnosed glioma patients: which symptom clusters are independently associated with functioning and global health status? Neuro Oncol 21(11):1447-1457. https://doi.org/10.1093/neuonc/noz118

35. Valko PO, Siddique A, Linsenmeier C, Zaugg K, Held U, Hofer S (2015) Prevalence and predictors of fatigue in glioblastoma: a prospective study. Neuro Oncol 17(2):274-281. https://doi.org/10. 1093/neuonc/nou127

36. Spielman AJ, Caruso LS, Glovinsky PB (1987) A behavioral perspective on insomnia treatment. Psychiatr Clin N Am 10(4):541553. https://doi.org/10.1016/s0193-953x(18)30532-x

37. Garland SN, Johnson JA, Savard J et al (2014) Sleeping well with cancer: a systematic review of cognitive behavioral therapy for insomnia in cancer patients. Neuropsychiatr Dis Treat 10:11131123. https://doi.org/10.2147/NDT.S47790

38. Schutte-Rodin SL, Broch L, Buysee D, Dorsey C, Sateia M (2008) Clinical guideline for the evaluation and management of chronic insomnia in adults. J Clin Sleep Med 4(5):487-504. https://doi.org/ $10.5664 / \mathrm{jcsm} .27286$

39. Daly FN, Schiff D (2007) Expert review of neurotherapeutics supportive management of patients with brain tumors supportive management of patients with brain tumors. Expert Rev Neurother 7(10):1327-1336. https://doi.org/10.1586/14737175.7.10.1327

Publisher's note Springer Nature remains neutral with regard to jurisdictional claims in published maps and institutional affiliations. 